\title{
Review of: "Mitonuclear interactions affect locomotor activity and sleep in Drosophila melanogaster"
}

Xingbo Zhao ${ }^{1}$

1 China Agricultural University

Potential competing interests: The author(s) declared that no potential competing interests exist.

This is interesting work, with different fly cybrid lines to check mtDNA effect. I suggest two additional analyses. One is for the nuclear genomes, which is the comparison between original line and the cybrid line, they share same mitogenome, but different nuclear genomes, what happened and how much changed on traits (i.e., actives or sleep). The other one is the comparisons among 8 mitoypes, what extent can be concluded on traits?

Small question: what are them for dot or dots on some blocks in Fig1, Fig2, Fig3, Fig4? and $\square$ what are As, Bs, Cs in all Figures? 